\title{
Bilateral incomplete Tapia's syndrome after surgical drainage, with orotracheal intubation, of an inflammatory process of the neck
}

\author{
Baroni $\mathrm{L}^{1}$, Zuliani $\mathrm{C}^{2}$ and Virgilio $\mathrm{C}^{3}$ \\ ${ }^{1}$ Primary Care Unit, Northern District, AULSS 9, Treviso (TV), Italy \\ ${ }^{2}$ Department of Neurology, General Hospital, Mirano (VE), Italy \\ ${ }^{3}$ Radiology Operative Unit - Mestre (VE), Italy
}

\begin{abstract}
Tapia's syndrome is characterized by unilateral paralysis of the tongue and vocal cord, with normal function of the soft palate, and is caused by lesions of the recurrent laryngeal branch of the X (vagus) and the XII (hypoglossal) cranial nerves. Parotid or other tumours, and infectious processes or traumatic injuries to the upper neck mainly cause it, but it can also occur, as a rare consequence of orotracheal intubation for general anaesthesia. Furthermore, head position, especially neck hyperextension, during these procedures, may play a significant role in determining its incidence.

It may also be a rare complication of other procedures.

We report the case of a patient who developed bilateral palsy of the hypoglossal nerve following drainage (done during general anaesthesia with orotracheal intubation) of a neck abscess, which had developed as a complication of left carotid endarterectomy (CEA).
\end{abstract}

\section{Introduction}

Tapia's syndrome, first described in 1904 by the Spanish otorhinolaryngologist (ORL) Antonio Garcia Tapia, is characterized by unilateral paralysis of the tongue and vocal cord, with normal function of the soft palate, and caused by lesions of the recurrent laryngeal branch of the X (vagus) and the XII (hypoglossal) cranial nerves [1]. According to the degree of damage of the nerves, different symptoms, such as hoarseness, dyspnoea, difficulty in tongue movement with dysarthria and dysphagia, can be present [2].

Parotid or other tumours, and infectious processes or traumatic injuries to the upper neck mainly cause it $[3,4]$, but it can also occur, as a rare consequence of orotracheal intubation for general anaesthesia. In the latter case, there is a general consensus linking this type of lesion to the pressure exerted by the inflated cuff of the tracheal tube during the intubation, which compresses the extracranial X and XII cranial nerves within the larynx and/or the pharynx, resulting in subsequent neuroapraxia [5,6]. Furthermore, head position, especially neck hyperextension, during these procedures, may play a significant role in determining its incidence [7].

Injury of these nerves may also be a rare complication of other procedures, including the use of the laryngeal mask airway (LMA), through a direct compression of the hypoglossal nerve beneath the jaw angle during mask ventilation [8,9], rhinoplasty or septorhinoplasty [10-12], extreme head positioning during shoulder surgery [5], cervical laminoplasty [13], cardiac surgery [14-16].

Diagnosis is based on the presence of concurrent paralyses and the exclusion of central or vascular causes. Treatment is supportive, with emphasis on empiric corticosteroids and dysphagia therapy, as the symptoms usually resolve spontaneously over a period of from a week to three-six months [17]. Although the majority of the cases are unilateral, bilateral Tapia's syndrome has also been reported [18].

We report the case of a patient who developed bilateral palsy of the hypoglossal nerve following drainage (done during general anaesthesia with orotracheal intubation) of a neck abscess, which had developed as a complication of left carotid endarterectomy (CEA).

\section{Case report}

A 72-year-old white-female referred to the Cardiovascular Department of Mestre-Venice (Italy), was affected by type 2 diabetes treated with insulin and essential hypertension. She had recently suffered for a right capsular lacunar stroke. Angiography revealed the presence of haemodynamically irrelevant kinking of the right internal carotid artery, but also of an asymptomatic left internal carotid artery stenosis $>70-80 \%$, for which she underwent left carotid endarterectomy (CEA) with Dacron patch, in general anaesthesia. Laboratory findings before and after surgery were unremarkable. With no postoperative complications reported on discharge, at the fifth day post-operative check-up, the presence of a moderate sized, left-sided, stable hematoma of the neck was noted. At ten days after surgery, the woman complained of neck pain and fever. Clinical examination was significant for a palpable mass draining purulent material. For this,

Correspondence to: Luciana Baroni, MD, Primary Care Unit, Northern District, AULSS 9, Treviso (TV), Italy, Tel: +39-333.200.41.22; Fax: +39-02.700.546.177; E-mail: luciana_baroni@yahoo.it; lbaroni@ulss.tv.it

Key words: hypoglossal nerve palsy, orotracheal intubation, tapia's syndrome Received: July 09, 2016; Accepted: July 25, 2016; Published: July 29, 2016 
other objective signs of surgical site infection, and increased serum inflammatory markers, she was re-admitted.

Computed tomography (CT-scan) of the neck showed: "On the left-side, a non-homogenous hypodense mass extends from the carotid artery and jugular vein posteriorly, the thyroid, larynx and hypopharynx medially, and the sternocleidomastoid muscle laterally, from the horizontal ramus of the mandible to the middle-third of the left thyroid lobe. It imprints the sternocleidomastoid muscle, which is unevenly thickened, the larynx and hypopharynx, which are deviated to right, and the thyroid gland. A peripheral enhancement border, with gaseous bubbles compatible with an abscess formation delineates after contrast injection". Laryngoscopy confirmed right tracheal deviation and a mild suffusion of the left lateral wall of the oropharynx and hypopharynx, with obliteration of the pyriform sinus. No alteration of the tongue and pharyngo-laryngeal motility was detected. The patient underwent orotracheal intubation for general anaesthesia, and then drainage of the abscess and regrafting with autologous saphenous vein patch, after removal of the infected Dacron patch. Microbiologic cultures isolated Streptococcus agalactiae, treated with specific antibacterial drugs. At the end of surgery, a while after extubation, the patient's blood pressure rose suddenly to $245 / 90 \mathrm{mmHg}$, and she developed right hemiparesis with aphasia. She was reintubated and transferred to the intensive care unit (ICU). Magnetic resonance imaging (MRI) of the brain showed a left fronto-insular and a parietal ischemic lesion. The day after admission to the ICU, after extubation the presence of a severe dysphagia and a tongue palsy were noted, whereas the movements of the pharynx and soft palate where found to be normal on ORL consult. The clinical conditions required the placement of a percutaneous endoscopic gastrostomy (PEG). The patient was then transferred to our Neurorehabilitation Care Unit, where neurological examination confirmed the presence of right upper limb paresis, expressive aphasia and bilateral palsy of the tongue, the latter more marked on the right side. A laryngoscopy confirmed the bilateral integrity of the recurrent laryngeal nerve.

The cervical CT-scan control showed the partial persistence of the inflammatory mass, as described by the radiologist: "Solid bilobed formation projects onto the lumen of the hypopharynx, from the right antero-lateral wall just above the epiglottic vallecula, spreading along the homolateral floor of the mouth. Contralaterally, a second small solid formation adheres to the pharyngo-epiglottic fold at the same level. A small amount of solid tissue -most likely scar tissue-, surrounds the large supra-aortic vessels in the left latero-cervical area" (Figure 1 , red circles). Concentric-needle electrode ( $25 \mathrm{~mm}$ in length, 0.03 $\mathrm{mm}$ in diameter) electromyography (EMG) analysis of the bilateral genioglossus muscle was also performed, revealing the presence of spontaneous fibrillations and positive sharp waves; no voluntary

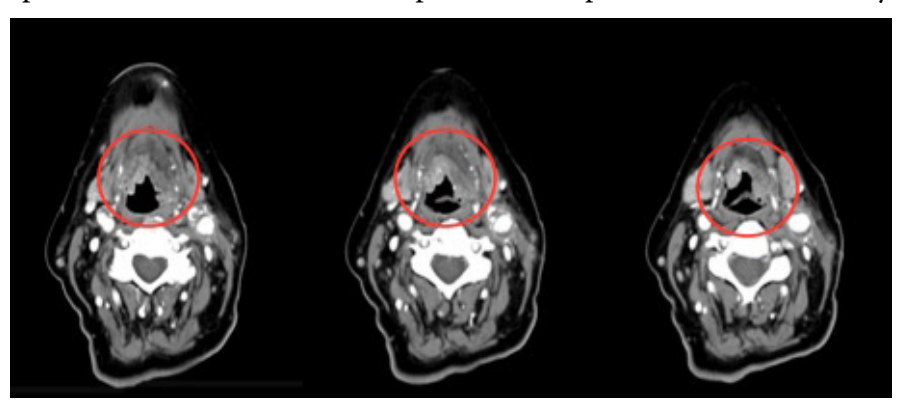

Figure 1. The neck TC-scan shows the presence of two solid bilobed formations, the first projecting onto the lumen of the hypopharynx, the second adhering to the pharyngoepiglottic fold at the same level. A small amount of solid tissue -most likely scar tissue-, surrounds the large supra-aortic vessels in the left latero-cervical area (red circles). contractile activity was detectable. This picture was compatible with complete denervation of these muscles, due to bilateral hypoglossal nerve tmesis. About ten days after steroid treatment the patient's tongue was retested by EMG, obtaining a recruitment pattern compatible with initial reinnervation on the right side of the tongue.

Unfortunately, the patient suddenly died from cardiogenic shock one month later. Autopsy was refused by the family.

\section{Discussion}

The hypoglossal nerve is a pure motor nerve that innervates all the muscles of the tongue. It is divided into the following segments: medullary, cisternal, skull base, nasopharyngeal and oropharyngeal, carotid space, and sublingual. Each segment can potentially be affected by different disorders [19]. Anatomically, the X and the XII cranial nerves lie near each other at the base of the tongue and in the pyriform fossa, and run on the lateral prominence of the anterior surface of the transverse process of the first cervical vertebra between the greater horn of the hyoid bone and the ligamentum stylo-hyoideum. The site where the nerves cross may be compressed by the endotracheal tube against these rigid anatomical structures, leading to a double-nerve injury. Moreover, hyperextension or excessive anterior-posterior and lateral flexion of the patient's head during surgery may also stretch or press these nerves between the tube and these hard tissues.

Atrophy or hypotrophy of tongue muscles, as consequence of denervation, is only seen when the nuclear or peripheral segments of the hypoglossal nerve are involved.

There are few reports in the literature of mono or bilateral palsy of the hypoglossal or laryngeal recurrent nerves after use of the LMA $[18,20,21]$. In this case the precise pathogenetic mechanism of nerve damage due to the use of laryngeal mask has not been determined, although erroneous positioning of the mask, excessive cuff pressure and particular positioning of the patient have been suggested. It is also possible that the duration of the mask location against the hypopharynx may be a factor.

Isolated unilateral or bilateral hypoglossal nerve damage, following transoral intubation, has also been reported [22-25]. The mechanism of injury is believed to be of neuropraxic origin, due to pressure on the lateral roots of the tongue during routine intubation with overextension of the head and the throat pack (tightly packed in the oro-pharynx), or also caused by a stretching of the nerve against the greater horn of the hyoid bone by the orotracheal tube [25].

Furthermore, some author reported recurrent laryngeal palsy following orotracheal intubation, likely linked to the compression by the cuff, of the anterior branch of inferior laryngeal nerve against the greater horn of the postero-medial part of the thyroid cartilage.

Friedrich et al. [26], estimated a $1.9 \%$ incidence of intubationrelated unilateral recurrent laryngeal nerve palsy, $1.4 \%$ transient and $0.5 \%$ permanent.

Moreover, cranial nerve injuries after CEA are described, especially for facialis, hypoglossus and vagus nerves; they can result from trauma during dissection, retraction, carotid clamping or local oedema [27,28], or finally rhinopharyngeal hematoma at the crossing of the vagus and hypoglossal nerves, due to the placement of a nasogastric tube [15].

Most of these injuries are believed to be of neuropraxic origin, as they are transient and with an excellent spontaneous recovery [7,29]. Therefore, the treatment is usually supportive, and a short course of steroids and vitamins may be used to accelerate the recovery period [30,31]. 
Our patient manifested clinical signs that helped us to localise the lesion to the peripheral segment of the XII cranial nerve, as confirmed by concentric-needle electrode electromyography (EMG) analysis, which showed the presence of total denervation of the genioglossus muscles bilaterally.

Based on clinical signs, EMG findings, and initial progressive recovery of lingual function up to death (occurred for other undercurrent diseases), our patient was diagnosed with bilateral incomplete Tapia's syndrome with isolated bilateral paralysis of the muscles of the tongue due to hypoglossal damage, without evidence of other affected cranial nerves.

There are very few case-reports similar to the present one, and all of them occurred after airway management in a surgical procedure with orotracheal intubation $[18,24]$. In conclusion, as Tapia's syndrome is a rare and possibly devastating condition, both anaesthesiologists and surgeons should be aware of the possible mechanisms of the injury and the importance of preventive measures. In fact, the incidence of the syndrome can be decreased by appropriate positioning of the head during surgery, early detection and correction of the head malposition, careful performance of the routine manoeuvres of airway management, appropriate flexion of orotracheal tube to prevent unperceived displacement, proper placement and amount of throat packing in the oropharynx, and a shortened intubation time.

In the current case, the clinical picture certainly cannot be directly ascribed to the surgical procedure of CEA, because of the time interval, but the lesion probably occurred as the result of a twofold compressive mechanism on the nerves: on one hand, eccentric compression by the orotracheal tube during anaesthesia; on the other hand, a prolonged concentric compression of the inflamed tissues of the neck. Although these potential factors may appear harmless in dayto-day practice, their simultaneous occurrence may have facilitated bilateral hypoglossal nerve injury in our patient. With a conservative management, including steroids and speech-swallowing rehabilitation, the patient had started to recover, before death happened.

\section{Acknowledgments}

The authors wish to thank Dr. Paul Griffith for his valuable suggestions and for the revision of the manuscript.

\section{References}

1. Schoenberg BS, Massey EW (1979) Tapia's syndrome. The erratic evolution of an eponym. Arch Neurol 36: 257-260. [Crossref]

2. Andrioli G, Rigobello L, Mingrino S, Toso V (1980) Tapia's syndrome caused by a neurofibroma of the hypoglossal and vagus nerves: case report. J Neurosurg 52: 730732.[Crossref]

3. Yavuzer R, Basterzi Y, Ozkose Z, YucelDemir H, Yilmaz M, et al. (2004) Tapia's syndrome following septorhinoplasty. Aesthetic Plast Surg 28: 208-211.[Crossref]

4. Cantalupo G, Spagnoli C, Cerasti D, Piccolo B, Crisi G, et al. (2014) Tapia's syndrome secondary to laterocervical localization of diffuse large cell Lymphoma. Brain Dev 36: 548-550. [Crossref]

5. Boisseau N, Rabarijaona H, Grimaud D, Raucoules-Aimé M (2002) Tapia's syndrome following shoulder surgery. Br J Anaesth 88: 869-870.[Crossref]

6. Dziewas R, Lüdemann P (2002) Hypoglossal nerve palsy as complication of oral intubation, bronchoscopy and use of the laryngeal mask airway. Eur Neurol 47: 239243.[Crossref]

7. Tesei F, Poveda LM, Strali W, Tosi L, Magnani G, et al. (2006) Unilateral laryngeal and hypoglossal paralysis (Tapia's syndrome) following rhinoplasty in general anaesthesia: case report and review of the literature. Acta Otorhinolaryngol Ital 26: 219-221. [Crossref]

8. Venkatesh B, Walker D (1997) Hypoglossalneuropraxia following endotracheal intubation. Anaesth Intensive Care 25: 699-700.[Crossref]
9. Agnoli A, Srauss P (1970) Isolated paresis of hypoglossal nerve and combined paresis of hypoglossal nerve and lingual nerve following intubation and direct laryngoscopy. HNO 18: 237-239. [Crossref]

10. Gevorgyan A, Nedzelski JM (2013) A late recognition of tapia syndrome: a case report and literature review. Laryngoscope 123: 2423-2427.[Crossref]

11. Lykoudis EG, Seretis K (2012) Tapia's syndrome: an unexpected but real complication of rhinoplasty: case report and literature review. Aesthetic Plast Surg 36: 557-559. [Crossref]

12. Ghorbani J, Dabir S, Givehchi G, Najafi M (2014) Co-presentation of Tapia's syndrome and pressure alopecia--A rare event after septorhinoplasty: A case report and literature review. Acta Anaesthesiol Taiwan 52: 38-40.[Crossref]

13. Lim KJ, Kim MH, Kang MH, Lee HM, Park EY, et al. (2013) Tapia's syndrome following cervical laminoplasty -A case report-. Korean J Anesthesiol 64: 172-174. [Crossref]

14. Nalladaru Z, Wessels A, DuPreez L (2012) Tapia's syndrome--a rare complication following cardiac surgery. Interact Cardiovasc Thorac Surg 14: 131-132.[Crossref]

15. Rotondo F, De Paulis S, Modoni A, Schiavello R (2010) Peripheral Tapia's syndrome after cardiac surgery. Eur J Anaesthesiol27: 575-576.[Crossref]

16. Sotiriou K, Balanika M, Anagnostopoulou S, Gomatos C, Karakitsos D, et al. (2007) Postoperative airway obstruction due to Tapia's syndrome after coronary bypass grafting surgery. Eur J Anaesthesiol 24: 379-379. [Crossref]

17. Tesei F, Poveda LM, Strali W, Tosi L, Magnani G, et al. (2006) Unilateral laryngeal and hypoglossal paralysis (Tapia's syndrome) following rhinoplasty in general anaesthesia: case report and review of the literature. Acta Otorhinolaryngol Ital 26: 219-221. [Crossref]

18. Stewart A, Lindsay WA(2002)Bilateral hypoglossal nerve injury following the use of laryngeal mask airway. Anesthesia 57: 264-265. [Crossref]

19. Thompson EO, Smoker WR (1994) Hypoglossal nerve palsy: a segmental approach Radiographics 14: 939-958.[Crossref]

20. Coninckx M, Cardoen S, Hemelsoet D (2015) Tapia's syndrome in the intensive care unit: a rare cause of combined cranial nerve palsy following intubation. ActaNeurolBelg 115: 533-537.[Crossref]

21. Sacks MD, Marsh D (2000) Bilateral recurrent laryngeal nerve neuropraxia following laryngeal mask insertion: a rare cause of serious upper airway morbidity. PaediatrAnaesth 10: 435-437. [Crossref]

22. Baumgarten V, Jalinski W, Bohm S, Galle E (1997) Hypoglossal paralysis after septum correction with intubation anaesthesia. Anaesthesist 46: 34-37. [Crossref]

23. Drouet A, Straboni JP, Gunepin FX (1999) [Paralysis of the hypoglossal nerve after orotracheal intubation for general anesthesia]. Ann Fr Anesth Reanim 18: 811-812. [Crossref]

24. Una E, Gandìa F, Duque JL (2009) Tongue paralysis after orotracheal intubation in a patient with primary mediastinal tumor. A case report. Cases $J$ 2: 9301. [Crossref]

25. Cinar SO, Seven H, Cinar U, Turgut S (2005) Isolated bilateral paralysis of the hypoglossal and recurrent laryngeal nerves (bilateral Tapia's syndrome) after transoral intubation for general anesthesia. Acta Anaesthesiol Scand 49: 98-99. [Crossref]

26. Friedrich T, Hansch U, Eichfeld U, Steinert M, Staemmler A, et al. (2000) Recurrent laryngeal nerve paralysis as intubation injury?. Chirurg 71: 539-544. [Crossref]

27. Maroulis J, Karkanevatos A, Papakostas K, Gilling-Smith GL, McCormick MS, et al. (2000) Cranial nerve dysfunction following carotid endarterectomy. IntAngiol 19: 237 241.[Crossref]

28. Sajid MS, Vijaynager B, Singh P, Hamilton G(2007) Literature review of cranial nerve injuries during carotidendarterectomy. Acta Chir Belg 107: 25-28. [Crossref]

29. Hung NK, Lee CH, Chan SM, Yh CC, Cherng CH, et al. (2009) Transient unilatera hypoglossal nerve palsy after orotracheal intubation for general anesthesia ActaAnaesthesiol Taiwan 7: 48-50. [Crossref]

30. Keane JR (1996) Twelfth-nerve palsy. Analysis of 100 cases. Arch Neurol 53: 561-566. [Crossref]

31. Boga I, Aktas S (2010) Treatment, classification, and review of Tapia syndrome. $J$ Craniofac Surg 21: 278-280.[Crossref]

Copyright: (C2016 Baroni L. This is an open-access article distributed under the terms of the Creative Commons Attribution License, which permits unrestricted use, distribution, and reproduction in any medium, provided the original author and source are credited. 